\title{
Conditioning Permeability Fields to Pressure Data
}

\author{
OLIVER, Dean S., Chevron Petroleum Technology Co. \\ HE, Nanqun, The University of Tulsa \\ REYNOLDS, Albert C., The University of Tulsa
}

Paper presented at the 5th European Conference on the Mathematics of Oil Recovery, Leoben, Austria, 3-6 Sept. 1996

\begin{abstract}
Generating realizations of the permeability field drawn from a probability density function conditioned on inaccurate pressure or saturation data is difficult, even if the unconditional realizations are Gaussian random fields, because the problem is highly nonlinear. Inefficient methods that generate large numbers of rejected images, such as simulated annealing, must be ruled out as impractical because of the repeated need for reservoir flow simulation.

In this paper, we present a two-step Markov chain Monte Carlo method of proposing transitions in the Metropolis-Hastings algorithm such that the resulting state has a high probability of acceptance. The first step is to propose an unconditional realization from a known probability distribution. This step could be carried out using any unconditional simulation technique so it is not limited to simple stochastic models. The second part of the proposed transition involves "history matching" of the unconditional simulation to production data that has noise added. The decision to accept or reject the resulting "history matched" realization is made on the basis of the MetropolisHastings algorithm. Using this procedure on a simple, but, highly nonlinear, problem results in a suite of realizations whose distribution is nearly indistinquishable from the analytical distribution. Because, however, calculation of the acceptance criterion for calibrated models is very difficult, we propose an approximate acceptance criterion in which all proposed transitions are accepted. This method is shown to work well on a small nonlinear problem for which the approximate distribution can be compared to the desired distribution.
\end{abstract}

\section{INTRODUCTION}

Calibration, in the groundwater field, or history matching, in petroleum engineering, is the process of adjusting a given permeability field so that, when it is used in a flow and transport simulator, it reproduces the measured head and concentration data. Sometimes, this is the only goal, although by starting from a realistic permeability distribution, one might hope to end up with a realistic distribution after calibration. In this sense, the resulting distribution represents a possible reservoir or aquifer model. If many of these models are generated and used to predict the probability of some future event, such as the concentration of a pollutant exceeding a critical value within a certain time span, then the reservoir models must be sampled from the space of all possible reservoir models, in such a way that the density of models reflects the probability density given by all the available data and geologic knowledge of the site.

The problem of generating an ensemble of conditional realizations is difficult for several reasons. First, the number of parameters is often very large so that techniques that work well for small problems are not suitable for reservoir characterization problems. Second, the connection between these parameters (typically permeability and porosity) and the observed data is highly nonlinear and can only be determined by solving a partial differential equation. Third, realistic models of the distribution of petrophysical properties tend to be quite complex.

When discussing previous work in this area, we find it useful to divide methods of simulation into two categories, those that add roughness or variability to a smooth estimate, and those that add a smooth correction to rough or variable fields of properties. The first method, adding variability to an optimal field, can be quite efficient if the relationship of the data to the model parameters is linear or nearly so. Ideally, one should then only have to solve the inverse or history matching problem once. For Gaussian random fields, generation of the rough field can sometimes be accomplished using a square-root decomposition of the a posteriori covariance matrix. Clifton and Neuman (1982) used this approach to simulate the 2D transmissivity field of the Avra Valley in Arizona. Oliver (1996a) applied the same idea to the generation of $2 \mathrm{D}$ realizations of the permeability field that were conditional to well test and interference test data by linearizing about the maximum a posteriori model. In neither case did the authors check to ensure that the distribution of realizations was close to the desired distribution. (It is not sufficient to establish that all the realizations satisfy the pressure data and the variogram.) More recently, Oliver et al. (1996) demonstrated the use of Markov chain Monte Carlo methods to ensure that the distri- 
bution of realizations would be assymptotically correct in the limit of an infinite collection of realizations. They found, however, that, because of the nonlinearity of the problem, the method becomes very inefficent when the permeability variation is large .

The second method, calibration of unconditional realizations, is an alternative that depends on the ability to calibrate efficiently. Chu et al. (1995) generated uncalibrated realizations from the prior distribution using the Cholesky method, then history matched the distribution to transient pressure data using a GaussNewton method. RamaRao et al. (1995) estimated the probability distribution of containment time for radioactive waste at the WIPP site by generating a large ensemble of "equally likely" transmissivity fields that agree with all measured data and can be considered plausible. Realizations of the transmissivity field that are conditional to measurements of transmissivity were generated using standard geostatistical techniques. The values of the transmissivity at "pilot points" (de Marsily et al., 1984) were then adjusted to calibrate the model to the pressure data. Although the problem of verifying that the generated realizations are indeed "equally likely" was discussed, verification was not attempted in this paper.

Another approach, that does not fall ințo either category, is to interpret well test data using analytical or semi-analytical tools. The results of the interpretation are parameters such as width of a channel or average permeability as a function of distance from the well. These interpretations are then used to condition realizations of the permeability field (Deutsch, 1992; Holden et al., 1995). One problem with this approach is that it limits the possible reservoir distributions to those for which simple analyses are possible. It also tends to oversimplify the relationship between model parameters and pressure data.

\section{SIMULATION AS MINIMIZATION}

Oliver (1996b) showed that conditional simulation of Gaussian random fields for linear problems could be approached as a minimization problem. When the covariance of the random field, $C_{M}$, and the variance of the measurements, $C_{D}$, are known, a conditional simulation can be obtained by minimizing the following objective function,

$$
\begin{aligned}
S(m)= & \left(m-m_{u s}\right)^{\mathrm{T}} C_{M}^{-1}\left(m-m_{u s}\right) \\
& +\left[G m-\left(d_{o b s}-C_{D}^{1 / 2} Z_{D}\right)\right]^{\mathrm{T}} C_{D}^{-1} \\
& \times\left[G m-\left(d_{o b s}-C_{D}^{1 / 2} Z_{D}\right)\right]
\end{aligned}
$$

where $m_{u s}$ is an unconditional realization of the model, the $i$ th row of $\mathrm{G}$ is the sensitivity coefficient for the $i$ th data point, and $C_{D}^{1 / 2} Z_{D}$ is a vector of normally distributed errors with variance $C_{D}$ that are subtracted from the observed data.

The procedure for linear problems is to create an unconditional realization of the model and an unconditional realization of the data. The model that minimizes Eq. (1) is a realization that is conditional to the observed data.

If the relationship of data to model parameters, $d=g(m)$, is not linear in $m$, and we wish to draw samples from the distribution

$$
\begin{aligned}
\pi(m) \propto & \exp \left\{-\frac{1}{2}(m-\mu)^{\mathrm{T}} C_{M}^{-1}(m-\mu)\right. \\
& \left.-\frac{1}{2}\left(g(m)-d_{o b s}\right)^{\mathrm{T}} C_{D}^{-1}\left(g(m)-d_{o b s}\right)\right\}
\end{aligned}
$$

then we might, by analogy with the linear problem, expect to obtain conditional realizations by calculating values of $m$ that minimize the following objective function,

$$
\begin{aligned}
S(m)= & \left(m-m_{u s}\right)^{\mathrm{T}} C_{M}^{-1}\left(m-m_{u s}\right) \\
+[g(m)- & \left.\left(d_{o b s}-C_{D}^{1 / 2} Z_{D}\right)\right]^{\mathrm{T}} C_{D}^{-1} \\
& \times\left[g(m)-\left(d_{o b s}-C_{D}^{1 / 2} Z_{D}\right)\right] .
\end{aligned}
$$

This is similar to the traditional regularized approaches to history matching, except that the regularization is with respect to the unconditional realization instead of a prior model, and the calculated data is matched to simulated data instead of observed data.:

\section{MARKOV CHAIN MONTE CARLO}

One way to ensure that the realizations that are generated are distributed correctly is to use the calibrated realizations as trial states in a Markov chain Monte Carlo (MCMC) method (see Tjelmeland et al., 1994; Oliver et al., 1996). In order, however, to use the Markov chain Monte Carlo method, we need to be able to calculate the probability of proposing the calibrated model. Recall that we first generate a new uncalibrated realization of the permeability field (it is not calibrated to the pressure data although it may be conditioned to other data) and a set of errors that will be added to the observed data. The state $m_{\text {cal }}$ that is proposed is the result of calibrating the uncalibrated realization to the simulated data (observed data plus noise) using Eq. (3). The joint probability density, $f\left(m_{u s}, d_{u s}\right)$, of proposing $\left(m_{u s}, d_{u s}\right)$ is easily calculated because $m_{u s}$ and $d_{u s}$ are independent random variables. Hence, for this problem,

$$
\begin{aligned}
f\left(m_{u s}, d_{u s}\right) & \propto \exp \left\{-\frac{1}{2}\left(m_{u s}-\mu\right)^{\mathrm{T}} C_{M}^{-1}\left(m_{u s}-\mu\right)\right. \\
& \left.-\frac{1}{2}\left(d_{u s}-d_{o b s}\right)^{\mathrm{T}} C_{D}^{-1}\left(d_{u s}-d_{o b s}\right)\right\}
\end{aligned}
$$

The joint probability density, $h\left(m_{c a l}, d_{u s}\right)$, of proposing $\left(m_{c a l}, d_{u s}\right)$ can, theoretically, be calculated if the functional relationship between $\left(m_{u s}, d_{u s}\right)$ and $\left(m_{\text {cal }}, d_{u s}\right)$ is known.

In our procedure we calculate $m_{c a l}$ using a GaussNewton method to find the minimum of Eq. (3), given 


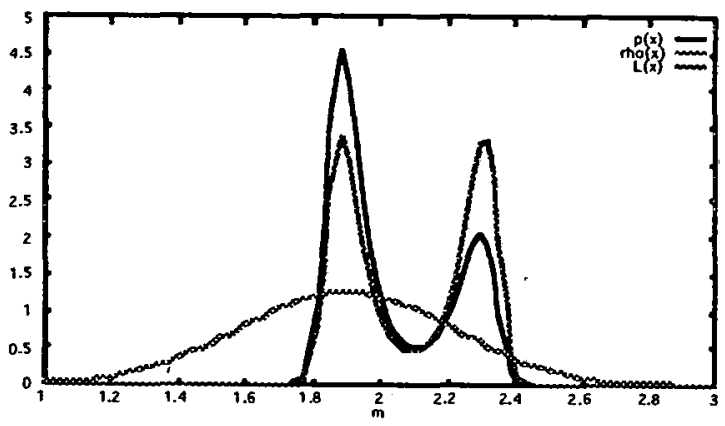

Figure 1: Prior probability, likelihood based on data, and a posteriori probability distributions for synthetic example.

$m_{u s}$ and $d_{u s}$. Reversing the procedure, we can instead solve for $m_{u s}$ as a function of $m_{c a l}$ and $d_{u s}$. If we exclude the regions of the $\left(m_{c a l}, d_{u s}\right)$ space that are inaccessible to the calibration routine, we obtain a unique one-to-one, invertable, relationship between $\left(m_{u s}, d_{u s}\right)$ and $\left(m_{c a l}, d_{u s}\right)$. The joint probability of proposing $\left(m_{c a l}, d_{u s}\right)$ can then be calculated as follows (see Wilks, 1962, sec. 2.8).

$$
h\left(m_{c a l}, d_{u s}\right)=f\left(m_{u s}, d_{u s}\right)|J|
$$

where $J$ is the Jacobian of the transformation, i.e.,

$$
J=\left|\frac{\partial\left(m_{u s}\right)}{\partial\left(m_{c a l}\right)}\right| .
$$

The probability of proposing $m_{c a l}$ is found by integrating $h\left(m_{c a l}, d_{u s}\right)$ over the data space.

$$
q\left(m_{c a l}\right)=\int_{D} h\left(m_{c a l}, d_{u s}\right) d d_{u s}
$$

For most practical problems, evaluation of the integral in Eq. (7) is to difficult to attempt. I will show a one-dimensional example for which the calculation can be performed, and I will show an approximation that seems to work well under a fairly broad range of conditions.

If the probability of proposing a transition to state $m_{j}$ is independent of the current state, Hasting's rule for the acceptance of a proposed transition from state $m_{i}$ to state $m_{j}$ can be written as

$$
\alpha_{i j}=\min \left\{1, \frac{\pi_{j} q_{i}}{\pi_{i} q_{j}}\right\}
$$

In our procedure, $q_{j}$ is the probability of proposing the calibrated model and depends only on the proposed state. The probability density for the calibrated model, $\pi_{j}$, is

$$
\begin{aligned}
\pi_{j} \propto & \exp \left\{-\frac{1}{2}\left(m_{j}-\mu\right)^{\mathrm{T}} C_{M}^{-1}\left(m_{j}-\mu\right)\right. \\
& \left.-\frac{1}{2}\left(g\left(m_{j}\right)-d_{o b s}\right)^{\mathrm{T}} C_{D}^{-1}\left(g\left(m_{j}\right) \div d_{o b s}\right)\right\}
\end{aligned}
$$

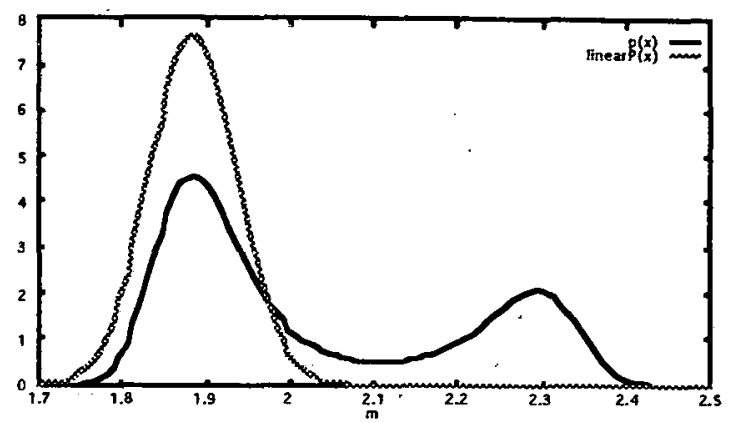

Figure 2: The a posteriori probability distributions for synthetic example and the linear approximation to the probability density based on the sensitivity at the maximum a posteriori point.

Note that the probability is not based on the quality of the match obtained in the minimization, but on the quality of the match to the prior model and the observed data.

\section{SYNTHETIC 1-D PROBLEM}

Because it is difficult to evaluate sampling procedures on large multivariate probability density functions, we first consider the simpler problem of sampling from the following univariate distribution

$$
\pi(m)=a \exp \left[-\frac{\left(m-m_{0}\right)^{2}}{2 \sigma_{m}^{2}}-\frac{(g(m)-d)^{2}}{2 \sigma_{d}^{2}}\right]
$$

where $m_{0}=1.9, d=0.8, \sigma_{m}^{2}=0.1, \sigma_{d}^{2}=0.01$, $g(m)=1-9(m-2 \pi / 3)^{2} / 2$, and $a \approx 4.567$. The parameters in this example were chosen to make it as close as possible to the example in Cunha (1996) without the additional complication of multiple minima outside the region of interest. The first term in Eq. (10) can be identified with an a priori probability distribution so we will use that terminology for it here. The other term is nonlinear and approximates the behavior of the likelihood term in the inverse problem. The a posteriori distribution, the a priori distribution, and the likelihood are all plotted in Figure 1.

One approach to the problem of sampling from $\pi(m)$ is to linearize the model-data relationship about the maximum a posteriori (MAP) point, which occurs at $m \approx 1.8805$, then propose models from the distribution based on the linearization, a Gaussian centered at the MAP point having a variance which satisfies the following relationship.

$$
\sigma_{M A P}^{-2}=\frac{G_{M A P}^{2}}{\sigma_{d}^{2}}+\frac{1}{\sigma_{m}^{2}}
$$

where $G_{M A P} \approx 1.893$ is the value of the derivative of $g(m)$, with respect to $m$, evaluated at the MAP value 
of $m$. Substituting numerical values into Eq. (11) gives $\sigma_{M A P}^{2} \approx 0.0027$. The Gaussian approximation to the a posteriori probability density is shown in Figure 2 along with the true probability density function.

In the method that we referred to as "global perturbations from the MAP," Oliver et al. (1996) proposed transitions from the current state (state $i$ ) to another state (state $j$ ) based on the linearized approximation to the nonlinear probability distribution, that is, the Gaussian probability density of Figure 2. Theory assures us that, even though the distribution that we sample from is quite unlike the true nonlinear distribution, the samples in the chain will (asymptotically) follow the true distribution as long as we use a correct acceptance criterion. Unfortunately, when Cunha (1996) used this approach to generate about five thousand states, she obtained a distribution that looked as if it were the result of sampling from a Gaussian probability density function centered on the MAP point. There was no hint of a secondary peak, or of any asymmetry to the distribution.

This result makes practical sense when we consider that the distribution we are proposing from is much narrower than the true distribution (see Figure 2) so that the probability of proposing a transition to a value of $m$ greater than 2.2, from the region of the MAP point, is approximately

$$
\begin{aligned}
q(m \geq 2.2) & =c \int_{2.2}^{\infty} \exp \left(-\frac{\left(m-m_{M A P}\right)^{2}}{2 \sigma_{M A P}^{2}}\right) d m \\
& \approx 6.610^{-10} .
\end{aligned}
$$

Clearly, it is unlikely that any states in the neighborhood of $m=2.3$ will be proposed in a chain of reasonable length. This seems inconsistent with the idea that the MCMC method, if it is truly sampling the distribution $\pi(m)$, should get about $33 \%$ of its samples from the neighborhood of the second peak. The explanation is that, although there is a very low probability for proposing a transition to the neighborhood of the secondary maximum, once it is proposed it will be accepted and the chain will almost certainly remain stuck at that state for another $10^{9}$ or so states. The chain will not just get stuck in the neighborhood of the secondary maximum, but rather it will remain stuck at a single state for a long period. Although many transitions to states in the neighborhood of the MAP will be proposed, the Metropolis-Hastings criterion gives a very low probability of acceptance to those transitions. While this behavior does serve to properly distribute the correct proportion of states within each interval, the distribution of realizations for chains of reasonable length will be very poor.

\section{CALIBRATE THE EXAMPLE}

For this simple, but highly nonlinear, problem it seems that the method of conditional simulation that adds variablity to an optimal estimate is a poor method of proposing states. In order to evaluate the alternative approach that we described previously, a simple Mathematica routine was written to implement the conditional simulation procedure based on calibration of unconditional simulations of the model.

Figure 3 shows all samples generated in the first 4000 trials. The distribution of states looks qualitatively reasonable and succeeding states do not appear to be correlated. The histogram of the frequency of occurrence of values from a 10,000 element chain shown in Figure 4 appears to match the true distribution quite well with the exception of a slight undersampling of elements in the region between the peaks.

We quantitatively compared the distribution of realizations with the true distribution using a chi-square test on the number of realizations within each decile. To do this I first calculated the values of $m$ corresponding to the decile points for the a posteriori distribution. Observed frequencies, by decile, are 1051, 1129, 1015, $1026,958,976,943,874,1015$, and 1013. Because the expected value within each decile is 1000 , the value of $\chi^{2}$ for this chain is 4.2 . With nine degrees of freedom, the probability of obtaining a $\chi^{2}$ value that large or larger is about $10 \%$, which indicates that the calibration algorithm did a good job of sampling from the a posteriori distribution.

Details of the calculation of the probability of proposing states for this particular problem can be found in Appendix A. Calculation of the Jacobian is the only difficult part of this procedure. If it is done accurately, the resulting distribution of states is very close to the desired distribution. It seems hopeless, however, to be able to accurately calculate the probability $q\left(m_{\text {cal }}\right)$ of proposing the calibrated states for large multivariate problems. Instead, we might evaluate the possibility that simple approximations, not requiring accurate calculation of the Jacobian, might result in reasonable distributions of states. Two possibilities suggest themselves. The first is to assume that $q_{i} / q_{j}=1$ and use the ratio of the probabilities of states for the acceptance criterion, i.e., $\alpha_{i j}=\min \left(1, \pi_{j} / \pi_{i}\right)$. The second is to assume that $q_{j}=\pi_{j}$ and simply accept all states that are the result of the calibration procedure. Figure 5 compares the distribution of states from the two approximations to the distribution that is expected from the a posteriori pdf. The simpler approximation (accepting all states) seems to result in a better distribution for this example. In particular, note that the fraction of states in the two peaks seems to be approximately correct. The main deficiency is an undersampling of states in the low probability region between the peaks. Whether or not this is important in practice is unknown. 


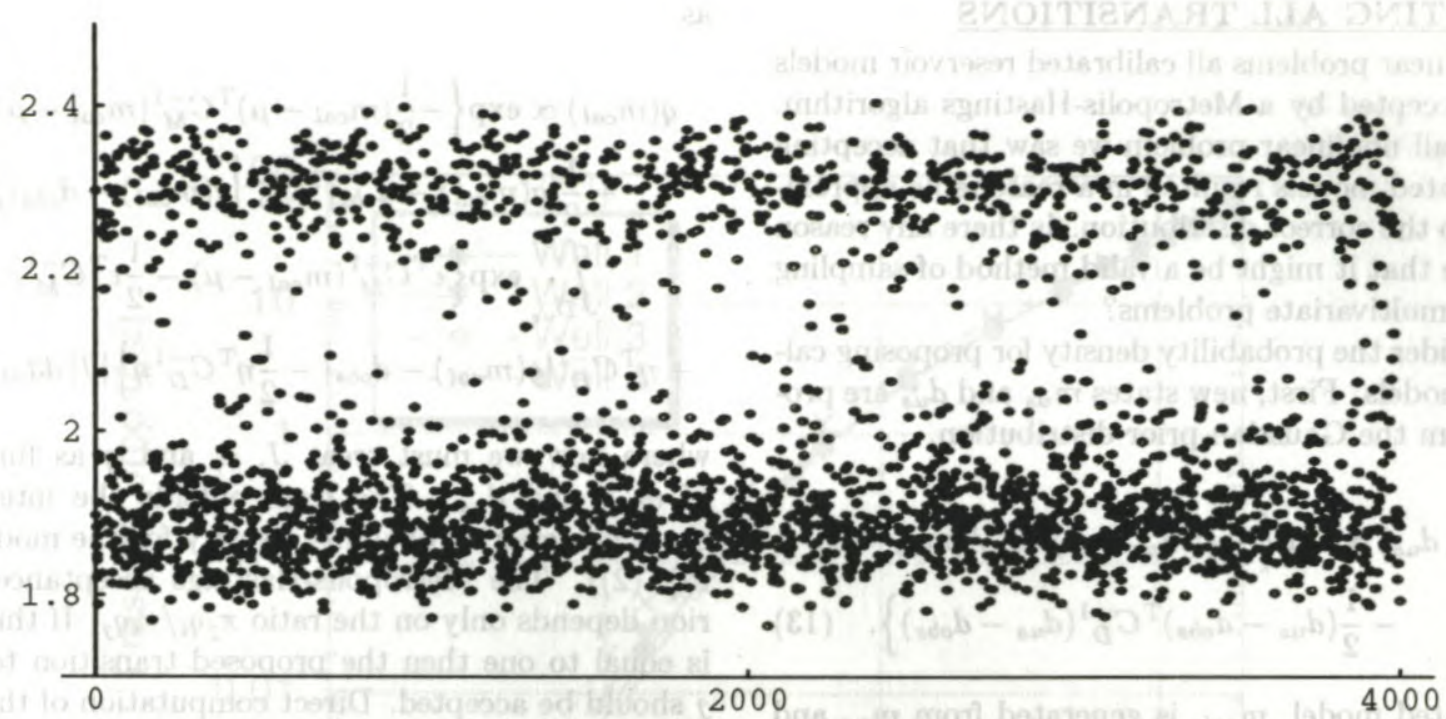

Figure 3: The Markov chain of states generated by "calibrating" unconditional simulations from the prior distribution to data.

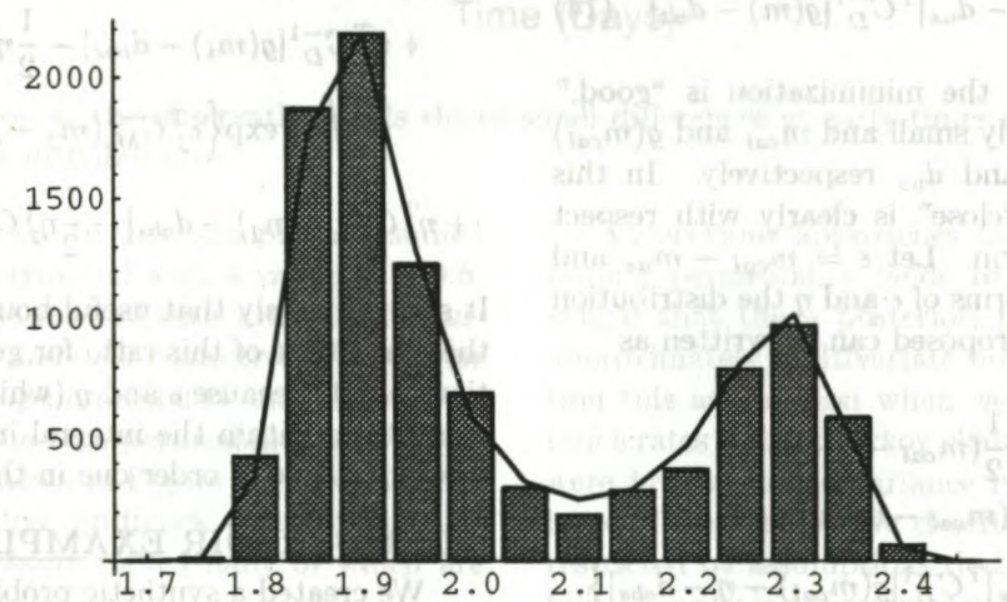

Figure 4: The frequency of occurrence of states within each frequency range seems to be a good approximation to the probability distribution that was to be sampled.
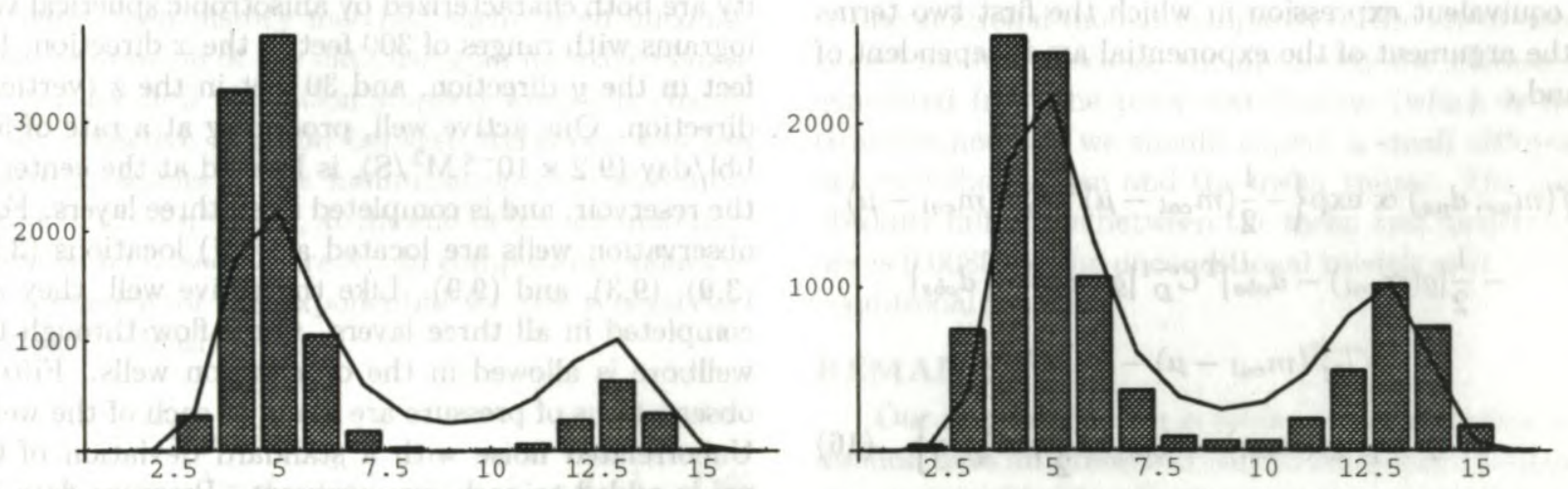

Figure 5: Two approximate methods for generating realizations from the nonlinear pdf. The acceptance criterion used for the distribution on the left involved only the ratio of the probability of successive states. For the histogram on the right, all transitions were accepted. 


\section{ACCEPTING ALL TRANSITIONS}

For linear problems all calibrated reservoir models will be accepted by a Metropolis-Hastings algorithm. For a small nonlinear problem we saw that accepting all calibrated models resulted in a reasonable approximation to the correct distribution. Is there any reason to believe that it might be a valid method of sampling for large multivariate problems?

Consider the probability density for proposing calibrated models. First, new states $m_{u s}$ and $d_{u s}$ are proposed from the Gaussian prior distribution,

$$
\begin{aligned}
f\left(m_{u s}, d_{u s}\right) & \propto \exp \left\{-\frac{1}{2}\left(m_{u s}-\mu\right)^{\mathrm{T}} C_{M}^{-1}\left(m_{u s}-\mu\right)\right. \\
& \left.-\frac{1}{2}\left(d_{u s}-d_{o b s}\right)^{\mathrm{T}} C_{D}^{-1}\left(d_{u s}-d_{o b s}\right)\right\} .
\end{aligned}
$$

A calibrated model, $m_{c a l}$, is generated from $m_{u s}$ and $d_{u s}$ by minimizing

$$
\begin{aligned}
O(m)=\left(m-m_{u s}\right)^{\mathrm{T}} C_{M}^{-1}\left(m-m_{u s}\right) \\
+\left[g(m)-d_{u s}\right]^{\mathrm{T}} C_{D}^{-1}\left[g(m)-d_{u s}\right]
\end{aligned}
$$

with respect to $m$. If the minimization is "good," $O\left(m_{c a l}\right)$ will be relatively small and $m_{c a l}$ and $g\left(m_{c a l}\right)$ will be close to $m_{u s}$ and $d_{u s}$ respectively. In this case, the meaning of "close" is clearly with respect to the weighted $L_{2}$ norm. Let $\epsilon=m_{c a l}-m_{u s}$ and $\eta=g\left(m_{c a l}\right)-d_{u s}$. In terms of $\epsilon$ and $\eta$ the distribution from which states are proposed can be written as

$$
\begin{aligned}
f\left(m_{u s}, d_{u s}\right) \propto & \exp \left\{-\frac{1}{2}\left(m_{c a l}-\epsilon-\mu\right)^{\mathrm{T}}\right. \\
& \times C_{M}^{-1}\left(m_{c a l}-\epsilon-\mu\right) \\
-\frac{1}{2}\left[g\left(m_{c a l}\right)-\right. & \left.\left.\eta-d_{o b s}\right]^{\mathrm{T}} C_{D}^{-1}\left[g\left(m_{c a l}\right)-\eta-d_{o b s}\right]\right\}
\end{aligned}
$$

where $m_{c a l}, \epsilon$, and $\eta$ must be thought of as functions of $m_{u s}$ and $d_{u s}$. Reorganization of the terms results in an equivalent expression in which the first two terms of the argument of the exponential are independent of $\eta$ and $\epsilon$.

$$
\begin{gathered}
f\left(m_{u s}, d_{u s}\right) \propto \exp \left\{-\frac{1}{2}\left(m_{c a l}-\mu\right)^{\mathrm{T}} C_{M}^{-1}\left(m_{c a l}-\mu\right)\right. \\
-\frac{1}{2}\left[g\left(m_{c a l}\right)-d_{o b s}\right]^{\mathrm{T}} C_{D}^{-1}\left[g\left(m_{c a l}\right)-d_{o b s}\right] \\
+\epsilon^{\mathrm{T}} C_{M}^{-1}\left(m_{c a l}-\mu\right)-\frac{1}{2} \epsilon^{\mathrm{T}} C_{M}^{-1} \epsilon \\
\left.+\eta^{\mathrm{T}} C_{D}^{-1}\left[g\left(m_{c a l}\right)-d_{o b s}\right]-\frac{1}{2} \eta^{\mathrm{T}} C_{D}^{-1} \eta\right\}
\end{gathered}
$$

The probability density for proposing a state, $m_{c a l}$, can be obtained by multiplying Eq. (16) by the Jacobian of the transformation between $m_{u s}$ and $m_{c a l}$, then integrating over the data space. We formally write this as

$$
\begin{array}{r}
q\left(m_{c a l}\right) \propto \exp \left\{-\frac{1}{2}\left(m_{c a l}-\mu\right)^{\mathrm{T}} C_{M}^{-1}\left(m_{c a l}-\mu\right)\right. \\
\left.-\frac{1}{2}\left[g\left(m_{c a l}\right)-d_{o b s}\right]^{\mathrm{T}} C_{D}^{-1}\left[g\left(m_{c a l}\right)-d_{o b s}\right]\right\} \\
\int_{D_{u s}} \exp \left\{\epsilon^{\mathrm{T}} C_{M}^{-1}\left(m_{c a l}-\mu\right)-\frac{1}{2} \epsilon^{\mathrm{T}} C_{M}^{-1} \epsilon\right. \\
\left.+\eta^{\mathrm{T}} C_{D}^{-1}\left[g\left(m_{c a l}\right)-d_{o b s}\right]-\frac{1}{2} \eta^{\mathrm{T}} C_{D}^{-1} \eta\right\}|J| d d_{u s}
\end{array}
$$

where now we must treat $J, \epsilon$, and $\eta$ as functions of $m_{c a l}$ and $d_{u s}$. The term outside the integral is the a posteriori probability density for the model (see Eq. (2)). The Metropolis-Hastings acceptance criterion depends only on the ratio $\pi_{j} q_{i} / \pi_{i} q_{j}$. If this ratio is equal to one then the proposed transition to state $j$ should be accepted. Direct computation of the ratio gives

$$
\begin{gathered}
\frac{\pi_{j} q_{i}}{\pi_{i} q_{j}}=\int_{D_{u s}} \exp \left\{\epsilon_{i}^{\mathrm{T}} C_{M}^{-1}\left(m_{i}-\mu\right)-\frac{1}{2} \epsilon_{i}^{\mathrm{T}} C_{M}^{-1} \epsilon_{i}\right. \\
\left.+\eta_{i}^{\mathrm{T}} C_{D}^{-1}\left[g\left(m_{i}\right)-d_{o b s}\right]-\frac{1}{2} \eta_{i}^{\mathrm{T}} C_{D}^{-1} \eta_{i}\right\}\left|J_{i}\right| d d_{u s} \\
\quad \div \int_{D_{u s}} \exp \left\{\epsilon_{j}^{\mathrm{T}} C_{M}^{-1}\left(m_{j}-\mu\right)-\frac{1}{2} \epsilon_{j}^{\mathrm{T}} C_{M}^{-1} \epsilon_{j}\right. \\
\left.+\eta_{j}^{\mathrm{T}} C_{D}^{-1}\left[g\left(m_{j}\right)-d_{o b s}\right]-\frac{1}{2} \eta_{j}^{\mathrm{T}} C_{D}^{-1} \eta_{j}\right\}\left|J_{j}\right| d d_{u s}
\end{gathered}
$$

It seems unlikely that useful bounds can be placed on the magnitude of this ratio for general nonlinear functions $g$ but, because $\epsilon$ and $\eta$ (which are small) occur in every term within the integral in Eq. (18), we expect the ratio to be of order one in the regions of interest.

\section{3-D RESERVOIR EXAMPLE}

We created a synthetic problem to investigate the feasibility of conditioning three-dimensional reservoir models to transient pressure data. The model consists of three $11 \times 11$ layers of gridblocks, each of which is 15 feet thick and 100 feet square. Permeability and porosity are both characterized by anisotropic spherical variograms with ranges of 300 feet in the $x$ direction, 180 feet in the $y$ direction, and 30 feet in the $z$ (vertical) direction. One active well, producing at a rate of 500 $\mathrm{bbl} /$ day $\left(9.2 \times 10^{-4} \mathrm{M}^{3} / \mathrm{S}\right)$, is located at the center of the reservoir, and is completed in all three layers. Four observation wells are located at $(i, j)$ locations $(3,3)$, $(3,9),(9,3)$, and $(9,9)$. Like the active well, they are completed in all three layers. Cross-flow through the wellbore is allowed in the observation wells. Fifteen observations of pressure are made at each of the wells. Uncorrelated noise with a standard deviation of 0.1 psi is added to each measurement. Pressure data for the observation wells (Figure 6) shows some variability among the wells at early times due to the nonuniform permeability and porosity fields. All the wells appear to have reached pseudosteady state flow by 0.45 days. 


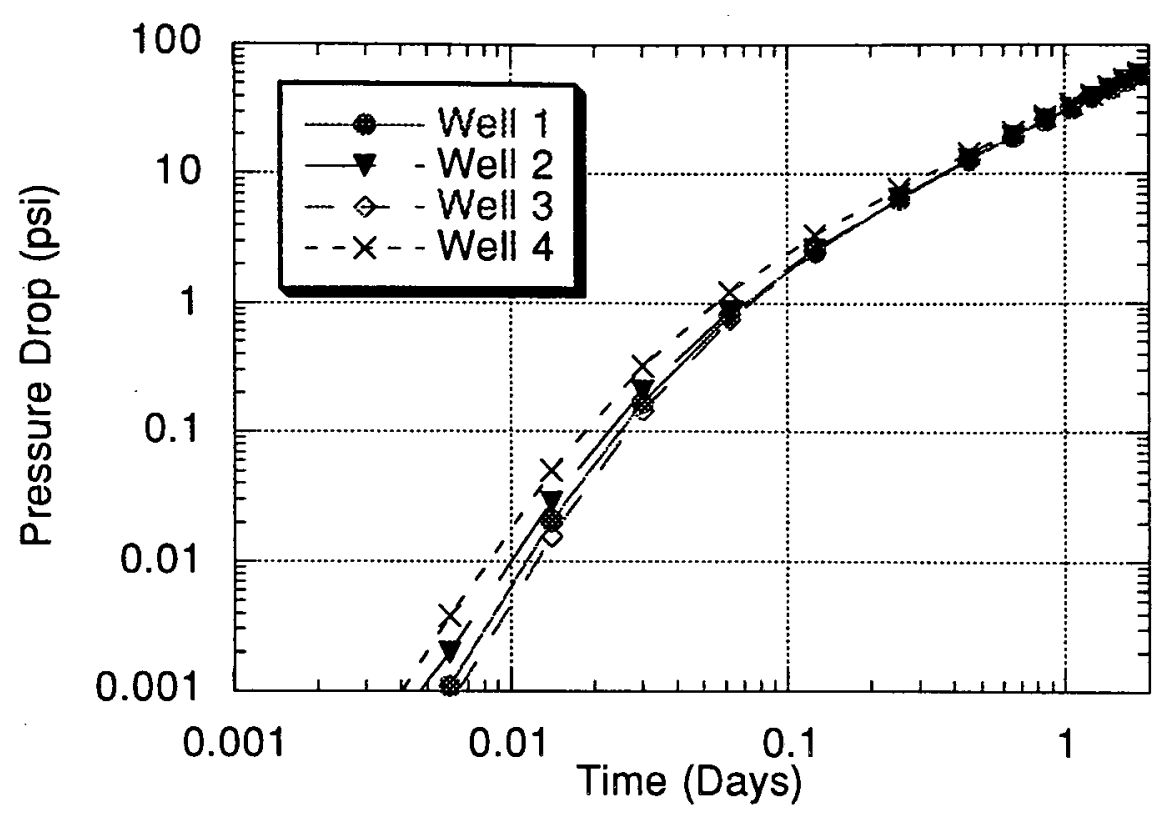

Figure 6: Pressure drop at the observation wells shows small differences at early times due to nonuniform permeability and porosity distribution.

Permeability (a scalar in this example) is assumed to be log-normally distributed with a variance of 0.6 . Porosity is normally distributed with a mean and standard deviation of 0.15 and 0.05 respectively. The prior correlation between $\log$-permeability and porosity is assumed to be 0.6 . The true permeability and porosity distributions are shown in Figure 7. There are 716 unknown, but correlated, gridblock parameters to be conditioned to 80 pressure data (many of which are redundant).

Fifty realizations each of the permeability and porosity fields were generated using the procedure described in this paper, that is, we started with unconditional realizations of the permeability and porosity fields, then history matched them to an unconditional realization of the data using an iterative GaussNewton method. Iteration stopped when the change in the objective function between iterations was less than 0.2. Values of the minimization objective function, $\epsilon^{\mathrm{T}} C_{M}^{-1} \epsilon+\eta^{\mathrm{T}} C_{D}^{-1} \eta$, at the end of history matching averaged 65.6 (see Figure 8). In comparison, values of the argument of the exponential for the a posteriori probability density,

$$
\begin{aligned}
& \left(m_{c a l}-\mu\right)^{\mathrm{T}} C_{M}^{-1}\left(m_{c a l}-\mu\right) \\
& \quad+\left(g\left(m_{c a l}\right)-d_{o b s}\right)^{\mathrm{T}} C_{D}^{-1}\left(g\left(m_{c a l}\right)-d_{o b s}\right),
\end{aligned}
$$

averaged 766 suggesting, for this example at least, that $\epsilon$ and $\eta$ are small enough that the approximate acceptance criterion may be valid.
In previous approaches to the problem of conditioning permeability fields to pressure data, we assumed that the a posteriori probability density was approximately multivariate normal. We had to question this assumption when we found that the acceptance rates in our Markov chain Monte Carlo methods were low when the variance in the permeability was large. The method we describe in this paper is not restricted by assumptions that the a posteriori probability density is approximately normal, but it is rather difficult to tell how far from normal the true distribution is. An indication of the non-normal nature of the distribution is seen by looking at the univariate statistics for the vertically averaged permeability in the set of unconditional models compared to the set of conditioned models. Because the unconditional models are generated from the prior distribution (which is multivariate normal) we should expect a small difference between the median and the mean values. The mean absolute difference between the mean and median values is 0.0085 for the unconditional models and 0.11 for conditional models.

\section{REMARKS}

Our approach to the problem of incorporating production data into reservoir characterization is to generate an ensemble of realizations of the permeability and porosity fields that are conditioned to flow and production data as well as static, linear data. It is important to us that the ensemble satisfy several criteria. First, 

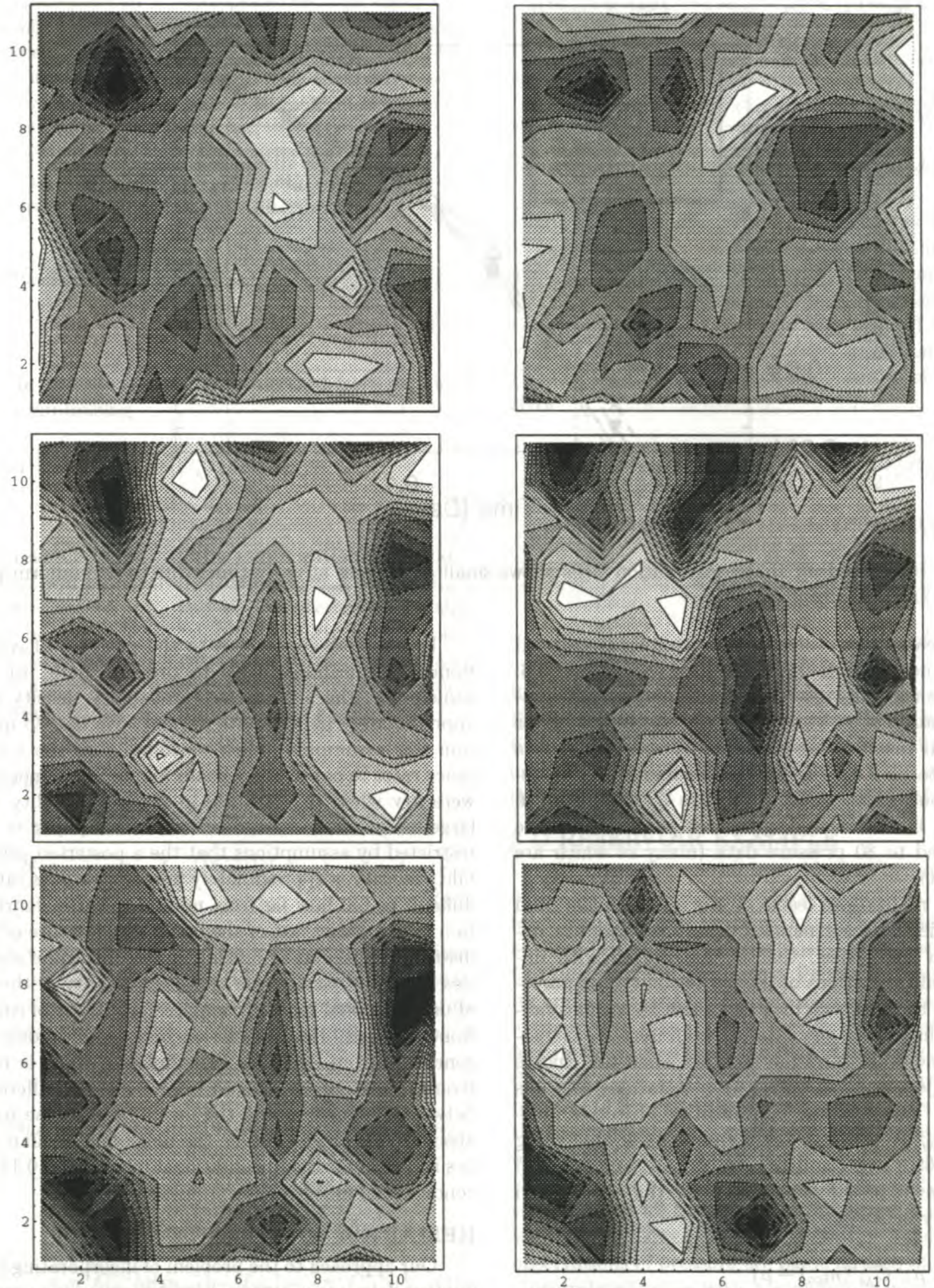

Figure 7: True log-permeability (left) and true porosity (right) by layer from the top of the reservoir (top) to the bottom. Darker shades of gray correspond to lower values. 


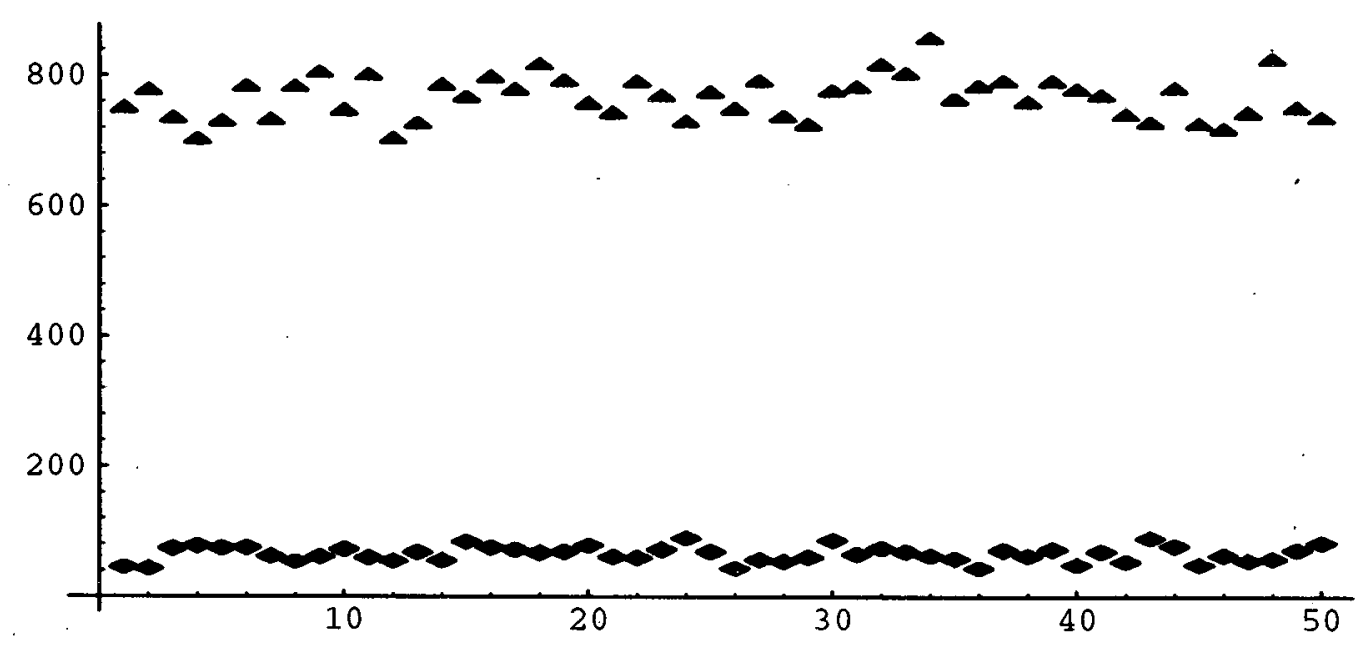

Figure 8: Points along the bottom of the figure are values of the objective function in Eq. (14) that was minimized in the history-matching procedure. Points along the top of the figure are values of the argument of the exponential part of the a posteriori probability distribution (Eq. (9)).

the frequency of occurrence of parameter values (permeabilities and porosities), within, an interval of values, must be approximately proportional to the conditional probability density for that parameter. Second, the space of acceptable models must be sampled efficiently, that is, we do not want many consecutive samples to be drawn from a small neighborhood of the acceptable models. Otherwise, predictions that are based on a finite set of realizations may grossly underestimate the true uncertainty in the reservoir. Further, because the relationship between the reservoir flow characteristics and the data is computationally complex, any method used to generate the suite of samples must be efficient in the sense of not generating large numbers of unacceptable models.

In this paper, we described a minimization method, much like traditional history-matching, that can be used to accomplish these objectives. The approximate method, in which every calibrated model is accepted as a new realization, is exact when the data is linearly related to the model parameters. We demonstrated that the approximate method worked well for a small, but highly nonlinear, model and discussed the reasons for believing that it is a satsifactory approach for large-scale reservoir characterization problems.

The efficiency of this method in practice will depend strongly on the efficiency of the history-matching algorithm because every realization will need to be history matched to production data. If, however, the volume of influence of the well test, or production data is fairly small, the history matching could be done on a small model extracted from the larger model.

\section{References}

Chu, L., Reynolds, A. C., and Oliver, D. S. (1995). Computation of sensitivity coefficients for conditioning the permeability field to well-test pressure data. In Situ, 19(2), 179-223.
Clifton, P. M. and Neuman, S. P. (1982). Effects of kriging and inverse modeling on conditional simulation of the Avra Valley aquifer in southern Arizona. Water Resour. Res., 18(4), 1215-1234.

Cunha, L. B. (1996). Sampling From the a Posteriori Probability Density Function for Permeability Fields Conditioned to Variogram and Well-Test Pressure Data. Ph.D. dissertation, University of Tulsa, Tulsa, Oklahoma.

de Marsily, G., Lavedan, G., Boucher, M., and Fasanino, G. (1984). Interpretation of interference tests in a well field using geostatistical techniques to fit the permeability distribution in a reservoir model. In G. Verly, M. David, A. G. Journel, and A. Marechal, editors, Geostatistics for Natural Resources Characterization, Part 2, pages 831-849. D. Reidell, Dordrecht, Holland.

Deutsch, C. V. (1992). Annealing Techniques Applied to Reservoir Modeling and the Integration of Geological and Engineering (Well Test) Data. Ph.D. thesis, Stanford University, Stanford, California.

Holden, L., Madsen, R., Skorstad, A., Jakobsen, K. A., Tjølsen, C. B., and Vik, S. (1995). Use of well test data in stochastic reservoir modelling, SPE 30591. In Proceedings from the Annual SPE Technical Conference, pages 653-659.

Oliver, D. S. (1996a). Multiple realizations of the permeability field from well test data. Soc. Petrol. Eng. J., 1(2), ???-???

Oliver, D. S. (1996b). On conditional simulation to inaccurate data. submitted to Math. Geology.

Oliver, D. S., Cunha, L. B., and Reynolds, A. C. (1996). A Markov chain Monte Carlo method of conditional simulation. Math. Geology. to appear. 
RamaRao, B. S., Venue, A. M. L., de Marsily, G., and Marietta, M. G. (1995). Pilot point methodology for automated calibration of an ensemble of conditionally simulated transmissivity fields 1 . Theory and computational experiments. Water Resour. Res., 31(3), 475-493.

Tjelmeland, H., Omre, H., and Hegstad, B. K. (1994). Sampling from Bayesian models in reservoir characterization. Technical Report Statistics No. 2/1994, The University of Trondheim.

Wilks, S. S. (1962). Mathematical Statistics. John Wiley \& Sons, New York. 644 p.

\section{APPENDIX A}

In this appendix we show a, few details related to the calculation of the Jacobian that was required for the 1-D problem. For every state in the Markov chain we draw an unconditional value of $m$ from the prior distribution, and an unconditional value of $d$ from the prior distribution for the data. We then use the value of $m$ that minimizes Equation (14) as a proposed value for the next state of the chain. In Figure 9 I have plotted the values of $m$ that minimize the energy functional versus the stochastic, unconditional, values of $m_{u s}$ and $d_{u s}$. Note that, when $d_{u s}$ is large, the calibrated value for $m$ will always be near $2 \pi / 3 \approx 2.094$, but, when $d_{u s}$ is small, values are obtained from the neighborhood of either peak.

To use the Metropolis-Hastings algorithm for sampling, we need to calculate the Jacobian of the transformation between $m_{c a l}$ and $m_{u s}$. Setting the gradient of the objective function in Eq. (3) to zero results in an equation for $m_{u s}$ as a function of $m_{c a l}$ and $d_{u s}$. Differentiation with respect to $m_{\text {cal }}$ gives

$\frac{\partial m_{u s}}{\partial m_{c a l}}= \begin{cases}0 & \text { forbidden region, } \\ \left.1+\frac{\sigma_{m}^{2}}{\sigma_{d}^{2}}\left[g^{\prime 2}+\left(g-d_{u s}\right) g^{\prime \prime}\right)\right] & \text { otherwise }\end{cases}$

where $g, g^{\prime}$, and $g^{\prime \prime}$ are evaluated at $m_{c a l}$. For a linear problem, the Jacobian would be a constant factor and there would be no forbidden region. Figure 10 shows, however, that the Jacobian for this nonlinear problem is far from constant. 


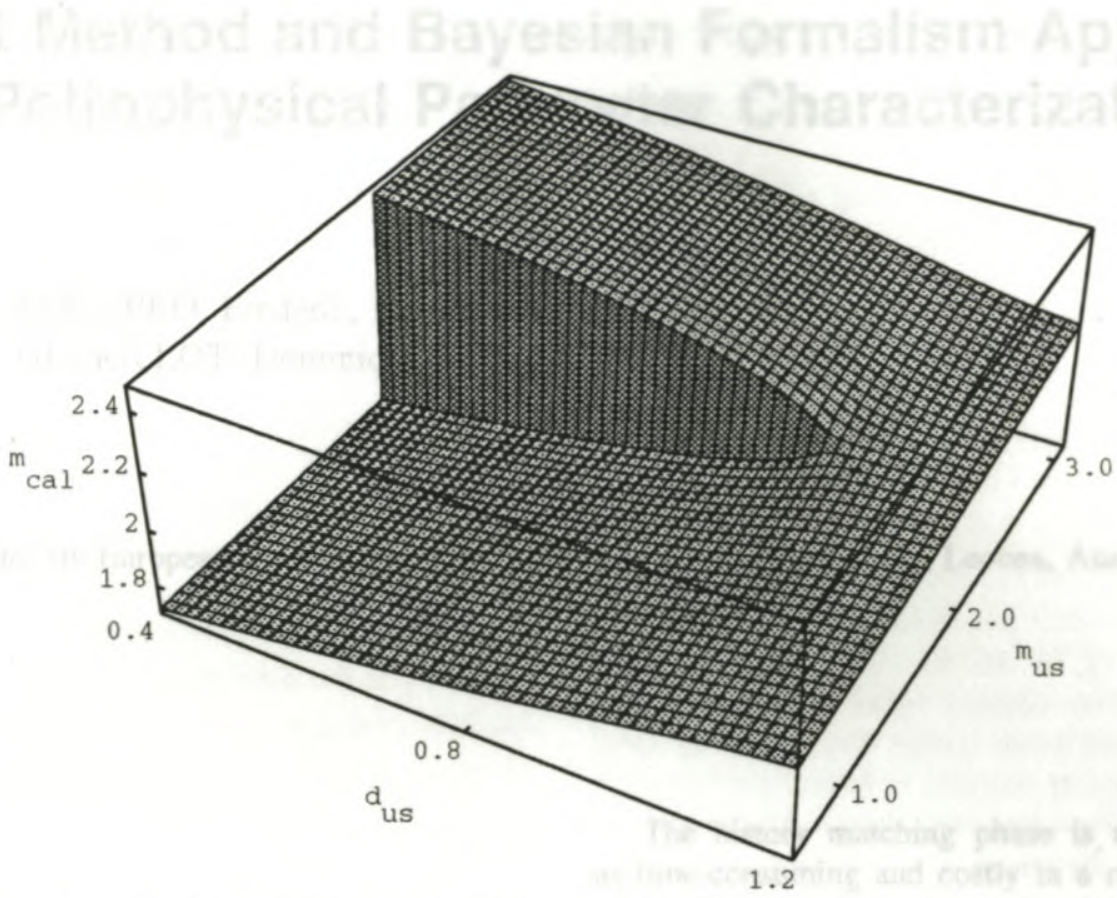

Figure 9: In the process of proposing a new state, we propose a value for $\mathrm{m}$ from the unconditional model distribution, and a value for $\mathrm{d}$ from the unconditional data distribution, then find a new model that minimizes a misfit. This figure shows the calibrated values of $\mathrm{m}$ as functions of simulated data and model values.

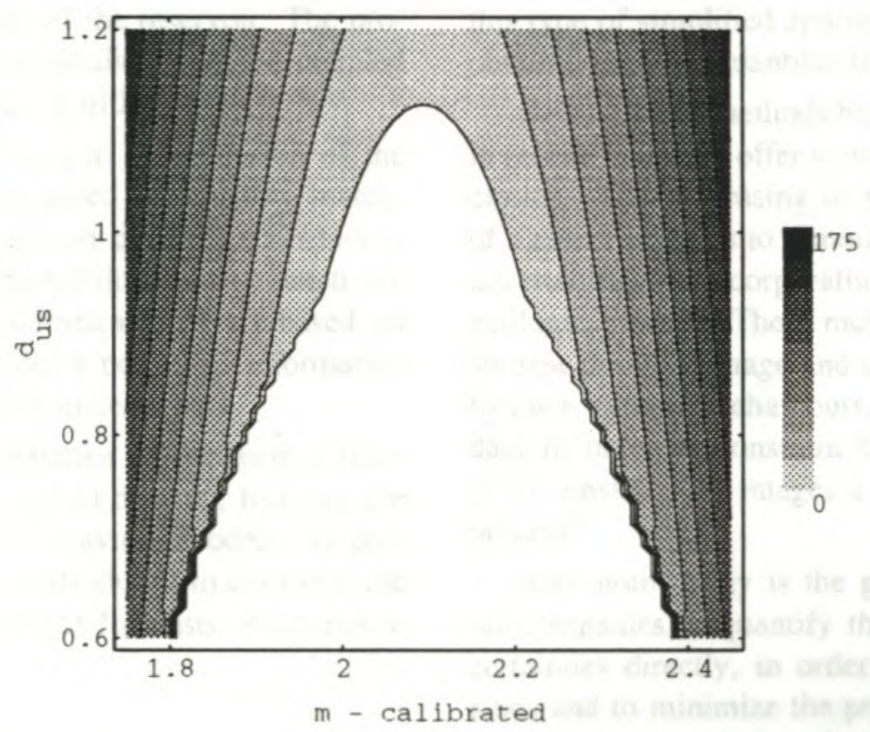

Figure 10: The Jacobian of the transformation between $m_{c a l}$ and $m_{u s}$. The flat region in the interior of the plot is inaccessible to the calibration algorithm. It corresponds to the discontinuity in Figure 9. 
